\title{
The betting market over time: Overround and surebets in European football
}

\author{
Carlos Gomez-Gonzalez* • Julio del Corral \\ Department of Economic Analysis and Finance, University of Castilla-La Mancha, Ciudad Real, Spain
}

Received: 27 April 2018

Revised: 11 October 2018

Accepted: 24 October 2018

\begin{abstract}
Sports betting is an important industry that generates billions of euros of revenue in Europe, and attracts the attention of sports economists. The recent entry of new companies could influence the market regarding the prices (overround), and hence, cross-bookmaker arbitrage opportunities. This paper investigates the movements of major betting companies in European football during the period from 2000-2001 to 2016-2017 with respect to these two issues. The results show a continuous decrease in the prices of the odds, which is especially noticeable in the top five leagues. In this line, the number of surebets have increased among major bookmakers in recent years, which offers bettors more possibilities of making a profit.
\end{abstract}

Keywords: betting; bookmakers; football; overround; surebets

JEL Classification Codes: L83, Z20

\section{Introduction}

Sports betting is a growing phenomenon that has become particularly relevant for sports economists. Although some countries hold legislative restrictions to gambling activities (Humphreys \& Pérez, 2012), the betting industry generates billions of euros of revenue, and involves the participation of a great number of agents in the European market. Only the UK online sports betting market was worth $£ 635$ million, and the biggest companies such as William Hill or Bet365 generated over a $£ 100$ million net revenues in 2011 (Gambling Compliance, 2012). Moreover, according to the estimations of the European Gaming and Betting Association, the industry is expected to increase its economic impact in the near future (EGBA, 2014).

The relationship between bettors and bookmakers, both interested in using all the information available to earn a profit, makes this market efficient (Sauer, 1998). Thus, although some works have identified potential biases, e.g., bettor sentiment (Levitt, 2004) or team's benefit (Paul \& Weinbach, 2009), multiple studies employ betting information to extract probabilities of winning and analyze team performance, e.g., Coates, Humphreys, and Zhou (2014) or

\footnotetext{
*Corresponding author. E-mail: carlos.gomez@uclm.es.

Citation: Gomez-Gonzalez, C., and del Corral, J. (2018) The betting market over time: Overround and surebets in European football, Economics and Business Letters, 7(4), 129-136.
} 
Humphreys, Paul, and Weinbach (2016). Besides the importance for academics, this market is interesting itself due to the growing volume of bets and bookmakers, which has definitively changed the business.

The overround, which is considered in the betting setting as the price of the odds, is a key factor in the market. In the fixed odds setting, this term refers to the profit margin of bookmakers, which is established by setting the odds in a way that the sum of the inverse is greater than one. The inverse of the odds represents the probability that the event occurs. In European football, bookmakers set the odds several days prior to the kick-off, and the odds remain available regardless of new information or betting volume (Forrest, Goddard, \& Simmons, 2005). The evolution of this market provides two interesting research opportunities.

First, the incorporation of new companies to the market overtime has influenced this overround. Consequently, monitoring the changes and analyzing the determinants is a relevant topic. Previous studies report a significant decrease of the overround in different sports leagues (Che, Feddersen, \& Humphreys, 2017; Lyócsa \& Fedorko, 2016). This study aims to analyze the evolution of the overround in major European football leagues in recent years and incorporate variables that help to understand the changes such as countries, divisions, bookmakers, or years. Moreover, the analysis focuses on major betting companies to understand their behavior in the market.

Second, the growing number of bookmakers and the evolution of the overround might have also boosted other phenomena in the betting market. The cross-bookmaker arbitrage has attracted attention in the literature on football betting since the contribution of Pope and Peel (1989), who demonstrated potential arbitrage opportunities in the English leagues across UK bookmakers. This concept is also known as surebet, and refers to the variation in the odds across bookmakers, which can generate the possibility of placing bets with no risk. Thus, bettors bet in each of the event's outcome in different bookmakers and earn a profit regardless of the final result. The potential decrease of the values of overround (reduced margins) and the growing number of betting companies (more dispersed odds) should generate more arbitrage opportunities.

Grant, Oikonomidis, Bruce, and Johnson (2018) note empirical studies that suggest a better coordination between bookmakers to avoid these opportunities, e.g., Dixon and Pope (2004), Deschamps (2008), or Vlastakis, Dotsis, and Markellos (2009). However, more recent results point a different direction, e.g., Franck, Verbeek, and Nüesch (2013) or Grant et al. (2018). The present study extends the years covered by previous studies and focuses on the existence of cross-bookmaker arbitrage among major companies only. We expect major companies to reduce arbitrage opportunities, which might have increased in recent years.

For this purpose, the analysis includes information from 16 football leagues in Europe and five important bookmakers during the period 2000-2001 to 2016-2017. The results help to extend the knowledge on the evolution of prices and margins that bookmakers use in the betting market of European football and the existence of surebets. Thus, the findings are relevant for bookmakers, bettors and analysists interested in the behavior of top companies in the market and cross-bookmaker arbitrage opportunities.

\section{Data and methods}

The analysis of the overround includes betting information from 16 football leagues in Europe, which has been collected from the website www.football-data.co.uk. This British website provides historical results and odds from different football leagues using the data from $w w w$.betbrain.com. In this study, the leagues are the first and second divisions of England, France, Germany, Italy and Spain, and the first divisions of Belgium, Greece, The Netherlands, Portugal, Scotland and Turkey. The database incorporates the odds from 5 important bookmakers: Bet365, Bwin, Interwetten, Ladbrokes, and William Hill. The complete sample includes 94,880 
football games over a 17-year period (2000-2001 to 2016-2017) ${ }^{1}$.

To calculate the overround embedded in the odds for each game, we follow Franck, Verbeek, and Nüesch (2010), who assume that the overround is as follows:

$$
\text { Overround }=\sum_{e} \frac{1}{o_{e}}
$$

Where $o_{e}$ are the decimal odds.

This information allows us to analyze the evolution of the prices overtime and compare bookmakers. Moreover, to further analyze the determinants of the overround, we use a regression model that includes the leagues, divisions, bookmakers and years as explanatory variables.

With respect to arbitrage, we also follow Franck et al. (2013) in order to identify cross-bookmaker opportunities. Specifically, we consider that an arbitrage opportunity arises when using the most favorable odd for each possible outcome (local win, draw, away win) and computing the overround, the value is lower than 0.99. Please, see an example. For a given game, bookmaker A offers 1.9, 2.4, 5.5 for home win, draw and away win, respectively. Bookmaker B offers 1.53, 3, 7 and bookmaker $\mathrm{C}$ offers $1.5,2.8,10$. The most favorable odds are 1.9 for local win in betting company A, 3 for draw in betting company B and 10 for away win in betting company $\mathrm{C}$. Computing the overround, we obtain the value 0.96 . Hence, a bettor could place a surebet in these three bookmakers.

\section{Results}

The price of the odds (overround), is a key factor in the remit of bookmakers. While bettors often try to find the best possible offer in the market, bookmakers use the information available to set accurate and competitive odds. Table 1 shows the average of the overround for all games by bookmaker during the analyzed period. By simply examining the aggregated average values, we corroborate that there are differences in the overround among bookmakers. The values range from 1.081 (Bet365) to 1.122 (Interwetten).

While Bet365 registered an average overround below 1.07 in 2009-2010, the majority of competitors offered values close to 1.10. The lower prices might have helped Bet365 to become the world's largest online gambling company with more than 22 million customers (Ahmed, 2017). The origin of Interwetten, which was founded in Austria in 1990, and actually based in Malta, might have attracted clients from areas with a higher purchasing power. This is a possible explanation for the higher prices offered by the company.

Table 1. Average overround by bookmaker.

\begin{tabular}{rrrrr}
\hline \hline Bet365 & Bwin & Interwetten & Ladbrokes & William Hill \\
\hline 1.081 & 1.091 & 1.122 & 1.104 & 1.103 \\
\hline \hline
\end{tabular}

Although Table 1 reports different values of overround among bookmakers, all of them have adjusted the margins in recent years. Figure 1 maps the evolution of the bookmakers' overround, which has progressively decreased during the analyzed period. This drop is especially important for some of the bookmakers such as Bet365, Ladbrokes, or William Hill since 2008. The new entry of competitors to the European football betting market, e.g., from Asia, have challenged the supremacy of UK companies and affected the efficiency of the market (Grant et al., 2018).

The leagues and divisions have also an influence on the overround. This is directly related to the information that bookmakers have available to set the odds. While more important leagues provide more information about the potential outcomes, low-impact leagues or second divisions

\footnotetext{
${ }^{1}$ The database only includes information for Bet365 since 2002-2003 and Bwin since 2004-2005.
} 
generate more uncertainty. Figure 2 shows the evolution of the overround by leagues and divisions over this 17-year period, which reveals two interesting results.

First, the value of the overround is lower in the most important leagues, i.e., first divisions of England, France, Germany, Italy and Spain. Only the Scottish Premiership shows similar overround values until the season 2008-2009. The British origin of some of the most important betting companies might help to explain this result. Then, the second divisions of the top five leagues always present higher values of overround than the first divisions. This result, which is consistent over time, is similar to minor impact leagues and is probably related to the media impact of the competition and the information available.

The influence of these variables on the overround is examined using a regression model. Table 2 displays the results.

Figure 1. Evolution of the average of the overround by bookmaker and market.
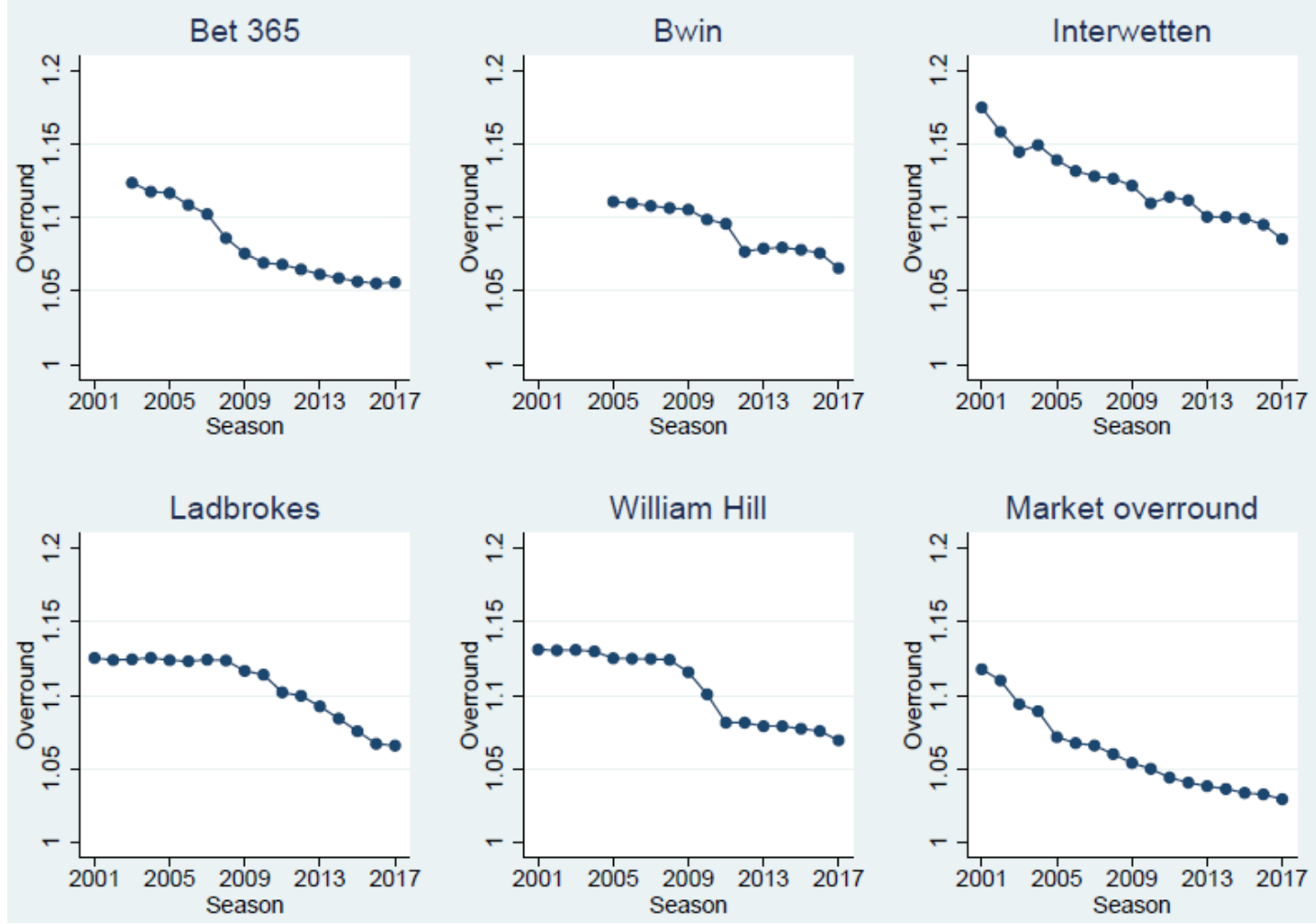

Figure 2. Average overround by country and league (2000-2001, 2008-2009, and 2016-2017).
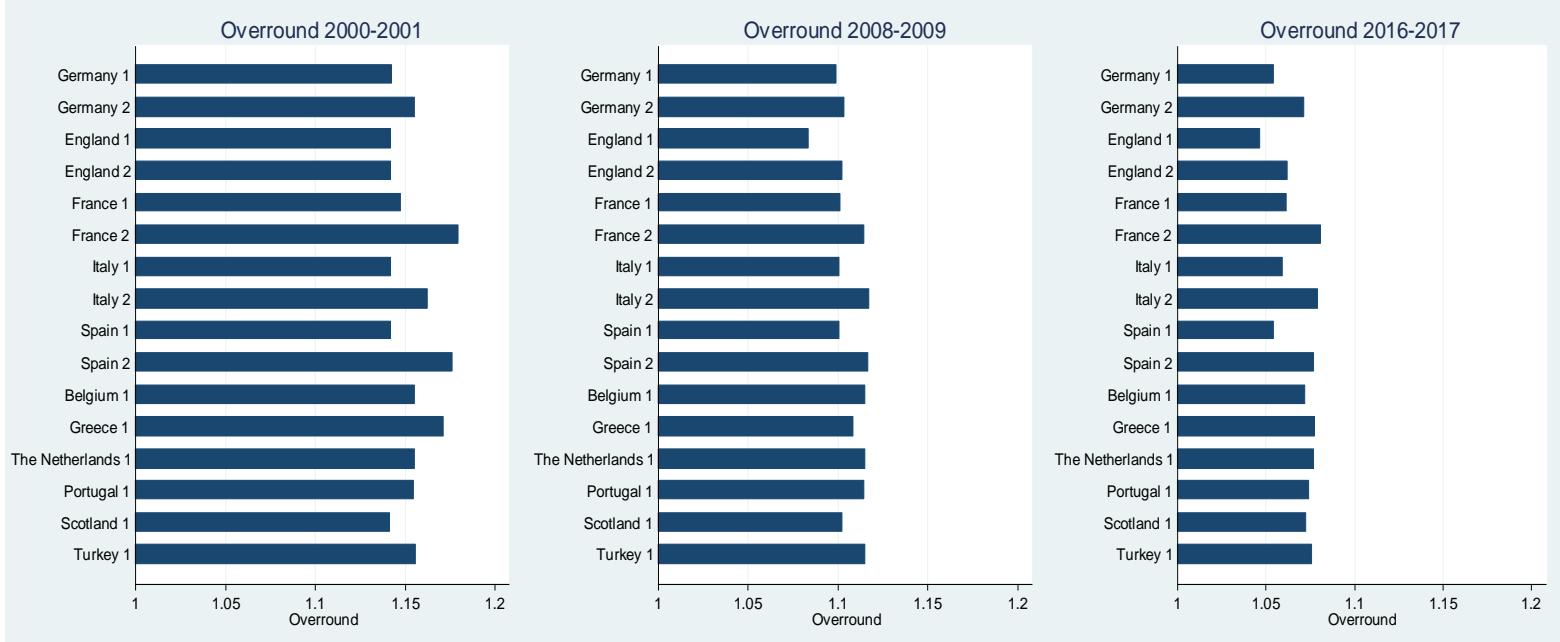
Table 2. Regression model estimates. Dependent variable: overround.

\begin{tabular}{|c|c|c|c|c|}
\hline Variable & Model 1 & Model 2 & Model 3 & Model 4 \\
\hline & Coef. & Coef. & Coef. & Coef. \\
\hline 2000-2001 (Omitted) & -- & -- & & \\
\hline 2001-2002 & $-0.009 * * *$ & $-0.009 * * *$ & & \\
\hline $2002-2003$ & $-0.011 * * *$ & $-0.018 * * *$ & & \\
\hline 2003-2004 & $-0.012 * * *$ & $-0.019 * * *$ & & \\
\hline 2004-2005 & $-0.020 * * *$ & $-0.027 * * *$ & & \\
\hline $2005-2006$ & $-0.023 * * *$ & $-0.030 * * *$ & & \\
\hline $2006-2007$ & $-0.026 * * *$ & $-0.032 * * *$ & & \\
\hline $2007-2008$ & $-0.030 * * *$ & $-0.037 * * *$ & & \\
\hline 2008-2009 & $-0.036 * * *$ & $-0.043 * * *$ & & \\
\hline 2009-2010 & $-0.044 * * *$ & $-0.051 * * *$ & & \\
\hline 2010-2011 & $-0.051 * * *$ & $-0.058 * * *$ & & \\
\hline 2011-2012 & $-0.056 * * *$ & $-0.063 * * *$ & & \\
\hline 2012-2013 & $-0.061 * * *$ & $-0.067 * * *$ & & \\
\hline 2013-2014 & $-0.063 * * *$ & $-0.069 * * *$ & & \\
\hline 2014-2015 & $-0.066 * * *$ & $-0.072 * * *$ & & \\
\hline $2015-2016$ & $-0.069 * * *$ & $-0.076^{* * *}$ & & \\
\hline 2016-2017 & $-0.075 * * *$ & $-0.081 * * *$ & & \\
\hline England (Omitted) & -- & & -- & \\
\hline Belgium & $0.009 * * *$ & & $0.009 * * *$ & \\
\hline France & $0.011 * * *$ & & $0.009 * * *$ & \\
\hline Germany & $0.006 * * *$ & & $0.004 * * *$ & \\
\hline Greece & $0.006 * * *$ & & $0.002 * * *$ & \\
\hline Italy & $0.011 * * *$ & & $0.008 * * *$ & \\
\hline The Netherlands & $0.009 * * *$ & & $0.008 * * *$ & \\
\hline Portugal & $0.007 * * *$ & & $0.007 * * *$ & \\
\hline Scotland & $0.001 * * *$ & & $0.001 * * *$ & \\
\hline Spain & $0.011 * * *$ & & $0.009 * * *$ & \\
\hline Turkey & $0.008 * * *$ & & $0.007 * * *$ & \\
\hline Top 5 league & $-0.016 * * *$ & & $-0.014 * * *$ & \\
\hline Interwetten (Omitted) & -- & & & -- \\
\hline Bet365 & $-0.037 * * *$ & & & $-0.041 * * *$ \\
\hline Bwin & $-0.022 * * *$ & & & $-0.031 * * *$ \\
\hline William Hill & $-0.018 * * *$ & & & $-0.019 * * *$ \\
\hline Ladbrokes & $-0.014 * * *$ & & & $-0.019 * * *$ \\
\hline Constant & $1.159 * * *$ & $1.150 * * *$ & $1.100 * * *$ & $1.122 * * *$ \\
\hline $\mathrm{R}^{2}$ & 0.786 & 0.549 & 0.061 & 0.217 \\
\hline Observations & 419,029 & 419,029 & 419,029 & 419,029 \\
\hline
\end{tabular}

Similar to the information from Figures 1 and 2, the model shows that the overround has significantly decreased over time. This is in line with previous studies in the literature that analyze English Premier League games over the seasons from 2004-2005 to 2011-2012 (Che et al., 2017), or ATP World Tour tennis games during the period 2000-2015 (Lyócsa \& Fedorko, 2016). The former argues that the growing number of new firms entering the market force traditional competitors to reduce prices. This is also true for the major betting companies analyzed in our sample.

Regarding the league and the division, we find interesting results. The overround in all nonTop 5 leagues is significantly higher than the overround in the English Championship League, except for the top five leagues. For instance, the overround in the leagues of Belgium and The Netherlands is 0.009 higher than in the English second division. In contrast, the overround in the Scottish league is only 0.001 higher. The British origin of some of the bookmakers may be 
related to this result. In any case, the overround in the first divisions of the top five leagues, is significantly lower (0.016). The impact (information and interest) that the top leagues generate has an influence on the bookmakers, who are forced to adjust the margins and reduce the overround. Lyócsa and Fedorko (2016) also find that more prestigious tennis tournaments (ATP1000 and Grand Slams) attract more bettors and reduce the margins of betting companies in the market.

Finally, regarding the differences among bookmakers, Interwetten is the most expensive company from our dataset in European football. This difference in the overround is about 0.037 with respect to Bet365 and 0.022 to Bwin. Besides the specific strategies that bookmakers might follow to set the odds, while trying to anticipate or influence betting volumes (Milliner, White, \& Webber, 2009), the country of origin may play a role. As Interwetten was founded in Austria in 1990 (currently based in Malta), this company might have been attracting bettors from countries with a higher purchasing power, e.g., Switzerland.

The evolution of the betting market in recent years is unlikely to have affected only the overround. Thus, the increasing number of companies in the market might have triggered changes in other factors such as arbitrage behaviours. Table 3 shows the numbers of surebets that have appeared in the different European football leagues over the analyzed period.

Table 3. Number of surebets by season and league.

\begin{tabular}{lrrrrrrrrrrrrrrrrr}
\hline \hline Season & $B 1$ & $D 1$ & $D 2$ & $E 0$ & $E 1$ & $F 1$ & $F 2$ & $G 1$ & $I 1$ & $I 2$ & $N 1$ & $P 1$ & SCO & SP1 & SP2 & T1 & Total \\
\hline $2000-2001$ & 0 & 0 & 0 & 0 & 0 & 0 & 0 & 0 & 1 & 0 & 0 & 0 & 0 & 0 & 0 & 0 & 1 \\
$2001-2002$ & 0 & 0 & 0 & 0 & 0 & 0 & 0 & 0 & 2 & 0 & 0 & 0 & 0 & 1 & 0 & 0 & 3 \\
$2002-2003$ & 0 & 0 & 0 & 0 & 0 & 1 & 0 & 0 & 0 & 0 & 0 & 0 & 0 & 2 & 0 & 0 & 3 \\
$2003-2004$ & 0 & 0 & 0 & 0 & 0 & 0 & 0 & 0 & 1 & 0 & 0 & 0 & 0 & 0 & 1 & 2 & 4 \\
$2004-2005$ & 1 & 0 & 0 & 0 & 0 & 0 & 0 & 0 & 0 & 0 & 0 & 0 & 0 & 0 & 2 & 1 & 4 \\
$2005-2006$ & 1 & 0 & 0 & 0 & 0 & 0 & 0 & 0 & 0 & 0 & 0 & 0 & 0 & 0 & 0 & 1 & 2 \\
$2006-2007$ & 0 & 0 & 0 & 0 & 0 & 0 & 0 & 0 & 1 & 5 & 0 & 0 & 0 & 1 & 0 & 0 & 7 \\
$2007-2008$ & 7 & 0 & 0 & 0 & 0 & 0 & 0 & 0 & 0 & 1 & 0 & 0 & 0 & 0 & 0 & 1 & 9 \\
$2008-2009$ & 0 & 0 & 0 & 0 & 0 & 0 & 1 & 2 & 0 & 1 & 0 & 0 & 0 & 0 & 0 & 0 & 4 \\
$2009-2010$ & 0 & 0 & 0 & 0 & 0 & 0 & 0 & 0 & 0 & 4 & 0 & 0 & 0 & 0 & 0 & 0 & 4 \\
$2010-2011$ & 0 & 0 & 0 & 0 & 0 & 0 & 2 & 0 & 0 & 0 & 0 & 0 & 0 & 0 & 0 & 0 & 2 \\
$2011-2012$ & 0 & 0 & 1 & 0 & 0 & 0 & 2 & 0 & 0 & 0 & 0 & 0 & 0 & 1 & 0 & 0 & 4 \\
$2012-2013$ & 0 & 8 & 0 & 0 & 1 & 1 & 0 & 4 & 0 & 0 & 1 & 1 & 0 & 2 & 1 & 1 & 19 \\
$2013-2014$ & 0 & 4 & 2 & 9 & 2 & 1 & 1 & 2 & 0 & 0 & 0 & 0 & 1 & 4 & 0 & 1 & 27 \\
$2014-2015$ & 0 & 8 & 2 & 5 & 0 & 3 & 2 & 1 & 0 & 2 & 1 & 1 & 3 & 0 & 1 & 0 & 28 \\
$2015-2016$ & 0 & 5 & 1 & 5 & 1 & 9 & 1 & 0 & 3 & 0 & 0 & 0 & 0 & 1 & 1 & 2 & 29 \\
$2016-2017$ & 0 & 9 & 3 & 18 & 0 & 10 & 3 & 1 & 11 & 0 & 1 & 1 & 1 & 15 & 0 & 3 & 75 \\
\hline Total & 9 & 34 & 9 & 37 & 4 & 25 & 12 & 10 & 19 & 13 & 3 & 3 & 5 & 27 & 6 & 12 & 225 \\
\hline \hline
\end{tabular}

Note: B1- Jupiler League, D1- Bundesliga 1, D2- Bundesliga 2, E0- English Premier League, E1- English Championship, F1- Ligue 1, F2- Ligue 2, G1- Superleague Greece, I1- Serie A, I2- Serie B, N1- Eredivisie, P1- Primeira Liga, SC0- Scottish Premier League, SP1- LaLiga1, SP2- LaLiga2, T1- Süper Lig.

The data reveals that the opportunities to place bets with no risk have greatly increased in recent years. While the 2011-2012 season only registered 4 surebets, the following seasons report numbers close to 30. Moreover, the last season shows a tremendous increase with respect to previous years -75 surebets. The top five leagues are the main responsible for the trend. For example, in the last season, bookmakers offered 15 and 18 surebets in the Spanish La Liga and the English Premier League, respectively.

The literature shows similar results, but with different subtleties. Franck et al. (2013) analyze the same major leagues during the period 2004-2005 to 2011-2012, and find 102 "intra-market" arbitrage opportunities. By reducing the number of betting companies and focusing on major bookmakers, we identify only 36 opportunities for this period. In the same line, Vlastakis et al. 
(2009) analyze five major bookmakers, but extended the sample of leagues up to 26 different countries. The authors find 63 cases of arbitrage in a two-year period. More recently, Grant et al. (2018) report a drastic increase in the number of surebets (545 cases in only 2,132 games from six major European football leagues in 2012-2013) due to a methodological change and the range of bookmakers included in the analysis. These authors use two types of bookmakers, i.e., position-takers and book-balancers, and develops a method that considers opportunities throughout the duration of the market offers, rather than at a single point in time. Thus, if an article includes a higher number of bookmakers, the range of the odds usually increases, so that arbitrage opportunities are more likely to happen.

By following previous studies of Vlastakis et al. (2009) and Franck et al. (2013), we aimed to investigate the number of surebets in recent years and exclusively among major bookmakers. The results show that there is an increasing number of arbitrage opportunities, even among the largest betting companies. This means that bettors do not need to create accounts in too many different small bookmakers or explore multiple sports competitions in order to find arbitrage opportunities in the market. A plausible explanation is that the reduction of the overround leads to a higher number of surebets. The reason behind this idea is that the lower the overround, the lower the range in the odds that is needed to generate a surebet. In an extreme case, when overround equals zero any difference in the betting odds lead to a surebet. The volume of bets is another possibility; however, in this study we do not have access to this information.

Future research can further explore the relationship between the origin of bookmakers and the overround of the leagues based in the same country. Similarly, the place of residence, and consequently the purchasing power of bettors, can have an influence on the prices set by bookmakers that receive their bets. Moreover, future efforts can point towards the number of surebets that have appeared in other betting settings over the last years.

\section{Concluding remarks}

The study focuses on the behavior of five major online sports betting companies with respect to the prices in the market and the existence of arbitrage opportunities. By analyzing 16 football leagues in Europe, we find that these betting companies have reduced the margins over the years. This is even more evident in the top five leagues, where the overround is significantly lower. The new entry of betting companies to the football betting market is the most likely cause.

Moreover, this study finds that cross-bookmaker arbitrage opportunities have increased in recent years, even among the largest companies. Therefore, the idea in the literature that bookmakers are more coordinated to avoid these failures at present finds no support in our analysis. These results are relevant for bettors, who do not need to register in multiple minor betting companies to find arbitrage opportunities, and for bookmakers in the market that need to prevent loses.

\section{Acknowledgements}

The authors acknowledge the valuable work of Mr. Jesús Miguel Gómez-Roso Jareño and Mr. Tomás Hidago Anguita in the data gathering process. We also gratefully acknowledge the comments received from two referees.

\section{References}

Ahmed, M. (2017) How UK beat the odds to win at online gambling, Financial Times, 12 August 2017, retrieved from https://www.ft.com/. 
Che, X., Feddersen, A., and Humphreys, B. R. (2017) Price setting and competition in fixed odds betting markets, in Rodríguez, P., Humphreys, B.R. and Simmons, R. (Eds): The Economics of Sports Betting, Cheltenham: Edward Elgar, 38-51.

Coates, D., Humphreys, B. R., and Zhou, L. (2014) Reference-dependent preferences, loss aversion, and live game attendance, Economic Inquiry, 52(3), 959-973.

Deschamps, B. (2008) Betting markets efficiency: Evidence from European football, Journal of Gambling Business and Economics, 2(1), 66-76.

Dixon, M.J. and Pope, P.F. (2004) The value of statistical forecasts in the UK association football betting market, International Journal of Forecasting, 20(4), 697-711.

EGBA (2014) Sports betting: Commercial and integrity issues, retrieved from http://www.egba.eu/.

Forrest, D., Goddard, J., and Simmons, R. (2005) Odds-setters as forecasters: The case of English football, International Journal of Forecasting, 21(3), 551-564.

Franck, E., Verbeek, E. and Nüesch, S. (2010) Prediction accuracy of different market structures-bookmakers versus a betting exchange, International Journal of Forecasting, 26(3), 448-459.

Franck, E., Verbeek, E. and Nüesch, S. (2013) Inter-market arbitrage in betting, Economica, 80(318), 300-325.

Gambling Compliance (2012) European regulated online markets, data report, retrieved from https://gamblingcompliance.com/.

Grant, A., Oikonomidis, A., Bruce, A. C., and Johnson, J. E. (2018) New entry, strategic diversity and efficiency in soccer betting markets: The creation and suppression of arbitrage opportunities, European Journal of Finance, 1-18.

Humphreys, B. R., and Pérez, L. (2012) Who bets on sports? Characteristics of sports bettors and the consequences of expanding sports betting opportunities, Estudios de Economía Aplicada, 30(2), 579-597.

Humphreys, B. R., Paul, R. J., and Weinbach, A. P. (2016) Performance expectations and the tenure of head coaches: Evidence from NCAA football, Research in Economics, 70(3), 482492.

Levitt, S. D. (2004) Why are gambling markets organised so differently from financial markets?, Economic Journal, 114(495), 223-246.

Lyócsa, Š., and Fedorko, I. (2016) What drives intermediation costs? A case of tennis betting market, Applied Economics, 48(22), 2037-2053.

Milliner, I., White, P., and Webber, D.J. (2009) A statistical development of fixed odds betting rules in soccer, Journal of Gambling, Business and Economics, 3(1), 89-99.

Paul, R. J., and Weinbach, A. P. (2009) Sportsbook behavior in the NCAA football betting market: Tests of the traditional and Levitt models of sportsbook behavior, Journal of Prediction Markets, 3(2), 21-37.

Pope, P. F., and Peel, D. A. (1989) Information, prices and efficiency in a fixed-odds betting market, Economica, 56(223), 323-341.

Sauer, R. D. (1998) The economics of wagering markets, Journal of Economic Literature, 36(4), 2021-2064.

Vlastakis, N., Dotsis, G. and Markellos, R. N. (2009) How efficient is the European football betting market? Evidence from arbitrage and trading strategies, Journal of Forecasting, 28(5), 426-444. 